\title{
Critical Issues in Mycobiota Analysis
}

\section{OPEN ACCESS}

Edited by:

David Berry,

University of Vienna, Austria

Reviewed by:

Carlotta De Filippo,

National Research Council, Italy

Micah Egge Dunthorn,

Kaiserslautern University of

Technology, Germany

*Correspondence:

Bettina Halwachs

bettina.halwachs@medunigraz.at

Gregor Gorkiewicz

gregor.gorkiewicz@medunigraz.at

Specialty section:

This article was submitted to

Microbial Symbioses,

a section of the journal

Frontiers in Microbiology

Received: 20 May 2016 Accepted: 24 January 2017 Published: 14 February 2017

Citation:

Halwachs B, Madhusudhan N Krause R, Nilsson $\mathrm{RH}$

Moissl-Eichinger $C$, Högenauer $C$,

Thallinger $G G$ and Gorkiewicz $G$ (2017) Critical Issues in Mycobiota

Analysis. Front. Microbiol. 8:180.

doi: 10.3389/fmicb.2017.00180

\section{Bettina Halwachs ${ }^{1,2,3 *}$, Nandhitha Madhusudhan ${ }^{1,2}$, Robert Krause $^{4}$, R. Henrik Nilsson ${ }^{5}$, Christine Moissl-Eichinger ${ }^{3,4}$, Christoph Högenauer ${ }^{2,3,6}$, Gerhard G. Thallinger ${ }^{3,7}$ and Gregor Gorkiewicz 1, 2, 3*}

${ }^{1}$ Institute of Pathology, Medical University of Graz, Graz, Austria, ${ }^{2}$ Theodor Escherich Laboratory for Medical Microbiome Research, Medical University of Graz, Graz, Austria, ${ }^{3}$ BioTechMed-Graz, Interuniversity Cooperation, Graz, Austria, ${ }^{4}$ Section of Infectious Diseases and Tropical Medicine, Department of Internal Medicine, Medical University of Graz, Graz, Austria, ${ }^{5}$ Department of Biological and Environmental Sciences, University of Gothenburg, Gothenburg, Sweden, ${ }^{6}$ Division of Gastroenterology and Hepatology, Department of Internal Medicine, Medical University of Graz, Graz, Austria, ${ }^{7}$ Institute of Molecular Biotechnology, Graz University of Technology, Graz, Austria

Fungi constitute an important part of the human microbiota and they play a significant role for health and disease development. Advancements made in the culture-independent analysis of microbial communities have broadened our understanding of the mycobiota, however, microbiota analysis tools have been mainly developed for bacteria (e.g., targeting the 16S rRNA gene) and they often fall short if applied to fungal marker-gene based investigations (i.e., internal transcribed spacers, ITS). In the current paper we discuss all major steps of a fungal amplicon analysis starting with DNA extraction from specimens up to bioinformatics analyses of next-generation sequencing data. Specific points are discussed at each step and special emphasis is placed on the bioinformatics challenges emerging during operational taxonomic unit (OTU) picking, a critical step in mycobiota analysis. By using an in silico ITS1 mock community we demonstrate that standard analysis pipelines fall short if used with default settings showing erroneous fungal community representations. We highlight that switching OTU picking to a closed reference approach greatly enhances performance. Finally, recommendations are given on how to perform ITS based mycobiota analysis with the currently available measures.

Keywords: microbiota, mycobiota, internal transcribed spacer (ITS), 16S rRNA gene, multiple sequence alignment (MSA), OTU picking, formalin-fixed paraffin-embedded tissue (FFPE), DNA isolation

\section{INTRODUCTION}

It is now well-established that the microbiota contributes significantly to human health and disease. So far, microbiota investigations have been mainly focused on bacteria, but also archea, viruses, and micro-eukaryotes such as protozoa and fungi are part of human-associated microbial communities. Fungi are prevalent in all microbially colonized body habitats including skin, the gastrointestinal (GI)-, urogenital-, and respiratory tract (Charlson et al., 2012; Findley et al., 2013; Hallen-Adams et al., 2015). Up to now more than 390 fungal species have been described in humans (Oever and Netea, 2014; Gouba and Drancourt, 2015). Depending on the habitat the abundance of fungal cells varies from $<0.1 \%$ of microorganisms in the GI tract to up to $10 \%$ on skin (Belkaid and Naik, 2013). An average fungal cell is about 100 -fold larger than an average bacterial cell, which translates into a significant fungal biomass, providing abundant bioactive molecules to the host and shaping its physiology (Underhill and Iliev, 2014). The GI mycobiota actively interacts with the immune system, for instance through the human innate immune receptor Dectin-1 able to dampen GI inflammation (Iliev et al., 2012). A balanced mycobiota prevents from hyperinflammation of the 
GI tract and alterations in fungal community composition due to antifungal drugs exacerbate colitis in mice (Wheeler et al., 2016). In humans genetic defects in certain immuneregulatory genes (e.g., STAT1, CARD9, etc.) or Il-17 and Il-22 signaling pathways lead to severe fungal syndromatic infections, such as chronic mucocutaneous candidiasis or the APECED (Autoimmune Polyendocrinopathy, Candidiasis, Ectodermal Dystrophy) syndrome (Oh et al., 2013; Underhill and Iliev, 2014). Compositional mycobiota shifts are reported in various diseases (Cui et al., 2013) and also interdependencies between the fungal and bacterial component of the microbiota exist. They are exemplified by disease-specific inter-kingdom alterations, reported for instance in inflammatory bowel disease (IBD, Ott et al., 2008; Hoarau et al., 2016; Sokol et al., 2016) or in the lung microbiome of cystic fibrosis patients (Kim et al., 2015). Importantly, fungi contribute significantly to human infections, especially in immune-compromised, chronically ill and intensive care patients wherein the respiratory or GI tract are often the origins of fungal systemic infections (Brown et al., 2012; Krause et al., 2016).

\section{INTERNAL TRANSCRIBED SPACERS (ITS) AS FUNGAL MOLECULAR BARCODES}

Currently, amplicon-based next generation sequencing is the standard measure for the culture-independent assessment of the mycobiota. Also metagenomic approaches are increasingly used, providing functional insights into the mycobiota. However, their broad application is still too costly due to the required sequencing effort to capture the relatively rare fungal biosphere and the special needs for bioinformatics analysis paired with underdeveloped fungal reference genome databases make metagenomics approaches still cumbersome (Tang et al., 2015). Early culture-independent mycobiota investigations used the eukaryotic 18S ribosomal RNA gene, in analogy to the prokaryotic $16 \mathrm{~S}$ rRNA gene, as molecular target enabling PCR amplification of fungal DNA and subsequent taxonomic profiling via sequence analysis (Simon et al., 1992; Kappe et al., 1996; Smit et al., 1999; Hunt et al., 2004). The 18S rRNA gene, however, is less discriminatory for fungi compared to its prokaryotic equivalent often failing to discriminate fungi at lower taxonomic levels, such as genus or species (Hartmann et al., 2010; Lindahl et al., 2013).

The prokaryotic and the eukaryotic rRNA operons exhibit different genetic architectures (Figures 1A,B). The eukaryotic rRNA cistron consists of the $18 \mathrm{~S}$ (small subunit, SSU), 5.8S, and $28 \mathrm{~S}$ (large subunit, LSU) rRNA genes transcribed as a unit by RNA polymerase I, including two internal transcribed spacer regions, ITS1 and ITS2, flanking the 5.8S rRNA gene. The two ITS regions are post-transcriptionally removed and are absent in the mature ribosome. Since they are dispensable for ribosome function, they experience a lower evolutionary pressure leading to higher sequence variability (Figures 1C-E). The increased level of sequence variability enables discrimination of even closely related taxa (e.g., at species level). In addition ITS sequences seem to represent superior molecular targets for fungal PCR amplification compared to SSU and LSU sequences, signified by higher positive PCR amplification rates (Schoch et al., 2012). Based on these observations, the Fungal Barcoding Consortium recently denoted the ITS region as the universal barcode for fungi superior to other molecular markers (Schoch et al., 2012).

In the following sections, we discuss the main steps of amplicon-based mycobiota analyses with special emphasis on the bioinformatics challenges emerging if standard bioinformatics analysis pipelines such as mothur, QIIME, or MICCA are employed (Schloss et al., 2009; Caporaso et al., 2010; Albanese et al., 2015).

\section{FUNGAL DNA ISOLATION}

A variety of studies have shown that DNA isolation methods and oligonucleotide primer choice significantly influence the outcome of molecular phylogenetic surveys (Gorkiewicz et al., 2013; Tedersoo et al., 2014; Hallen-Adams et al., 2015). Numerous protocols and kits are available for isolation of fungal DNA and they follow similar basic principles with slight modifications dependent on the specimen type used (Paulino et al., 2006; Ghannoum et al., 2010; Findley et al., 2013; Lindahl et al., 2013; Gosiewski et al., 2014; Oh et al., 2014). The basic protocol involves mechanical cell disruption using bead beating, followed by enzymatic cell lysis. Especially the addition of lyticase, and endoglucanase hydrolyzing the covalent bounds between $\beta$-(1-3)-D-glucose molecules in the fungal cell-wall glycan, is an essential step to enable complete fungal cell lysis (Muñoz-Cadavid et al., 2010; Goldschmidt et al., 2014). The final DNA purification step is often performed by using membranebased procedures (van Burik et al., 1998; Lindahl et al., 2013).

Aside of typically sampled native material (e.g., swabs, etc.) also other resources for mycobiota investigations exist. Formalinfixed paraffin-embedded (FFPE) tissue samples play an important role in the clinical context. Biopsies or surgically removed tissues are typically fixed in formalin (10\%) immediately after they are collected from the patient, thus they represent a well-preserved resource for the analysis of biomolecules including nucleic acids (Sangoi et al., 2009; Kocjan et al., 2015). FFPE specimens are typically used for diagnostic purposes (e.g., histopathology) but are also amenable for molecular scientific investigations. Their prevalence in biological repositories such as biobanks make them ideal specimens to study the mycobiota in the context of human disease (Yuille et al., 2008). About 70 commercially kits are available for DNA extraction out of FFPE material (Kocjan et al., 2015), however, nucleic acid isolation from FFPE material is challenging. Biomolecules are typically cross-linked and fragmented due to formalin, and factors such as the $\mathrm{pH}$ of the fixative, duration of fixation, and importantly the DNA extraction method applied greatly influence the quality of the extracted DNA (Bonin and Stanta, 2013; Kocjan et al., 2015). Factors such as residual formalin inhibiting proteinase $\mathrm{K}$ activity and omitting complete cell lysis, as well as the presence of PCR inhibitors in the DNA extract might altogether interfere with successful fungal DNA amplification (Coura et al., 2005; Muñoz-Cadavid et al., 2010). 
A

Prokaryotic rRNA operon

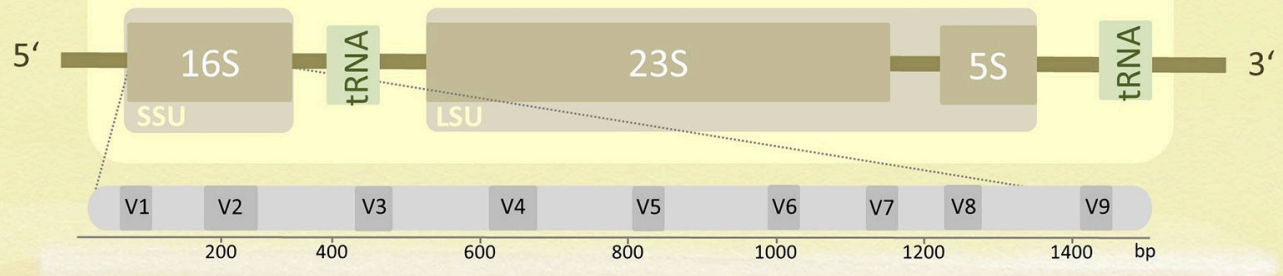

B

Eukaryotic rRNA operon
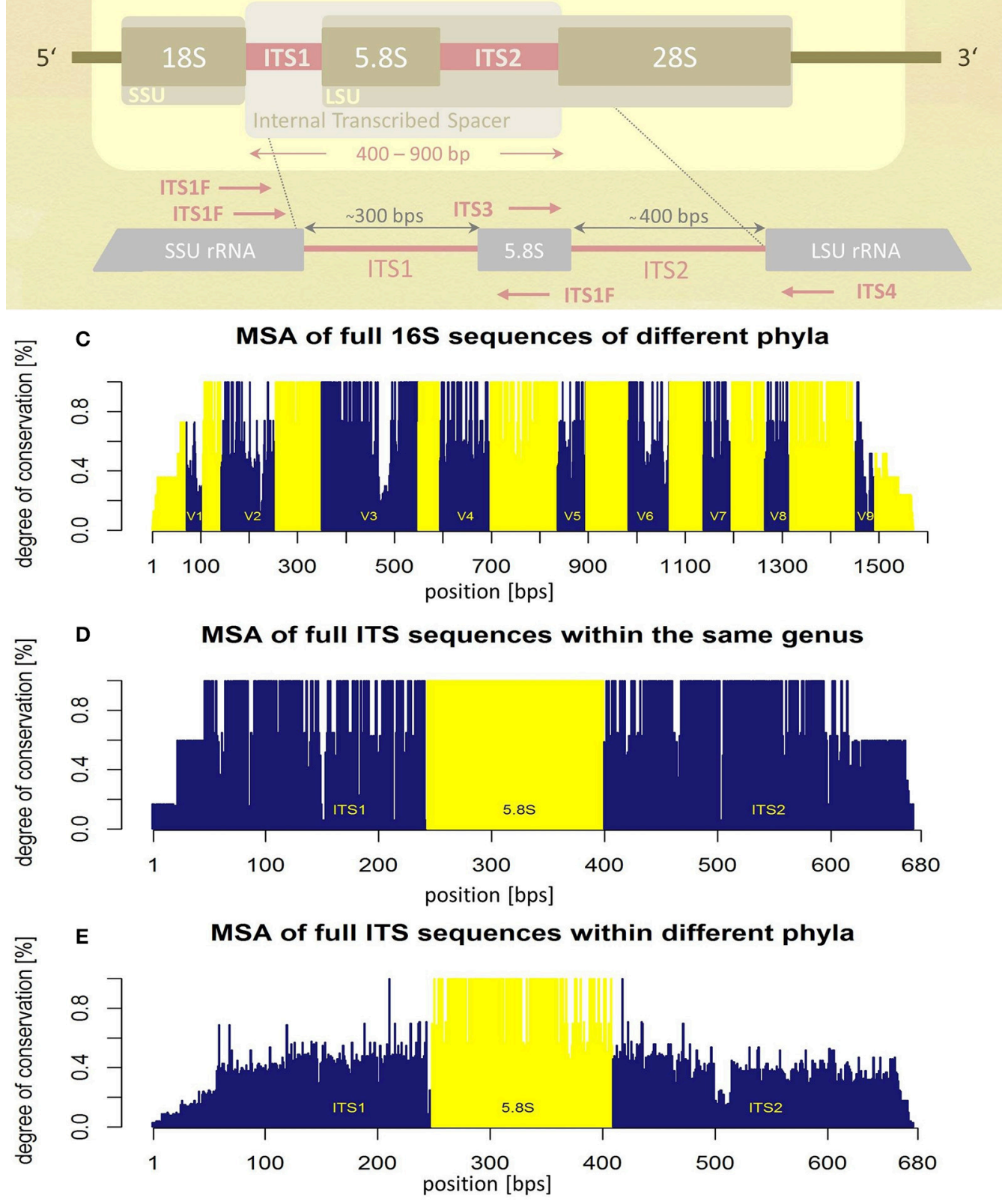

FIGURE 1 | Schematic representations of rRNA operons and their variability assessed by multiple sequence alignments (MSA). (A) Prokaryotic and (B) eukaryotic rRNA operons. Position and orientation of oligonucleotide primers used for ITS amplification are schematically indicated (for sequence information see Table 1). SSU, small subunit; LSU, large subunit; tRNA, transfer RNA; V1-V9, variable regions; ITS, internal transcribed spacer; bps, base-pairs. (C) Multiple 


\section{FIGURE 1 | Continued}

sequence alignment (MSA) of the entire 16S rRNA operon of five different bacterial species (encompassing five different phyla). Variable regions (V1-V9) are highlighted in blue, conserved regions in yellow, positions according to the E. coli 16S rRNA (GenBank acc. no.: J01695.2). (D) MSA of the complete internal transcribed spacer region of five different fungal species of the same genus (Hydnum sp.). (E) MSA of the complete ITS region of seven fungal taxa representing different phyla. Information about sequences used for MSA generation (C,D) is given as Supplementary Tables S3-S5.
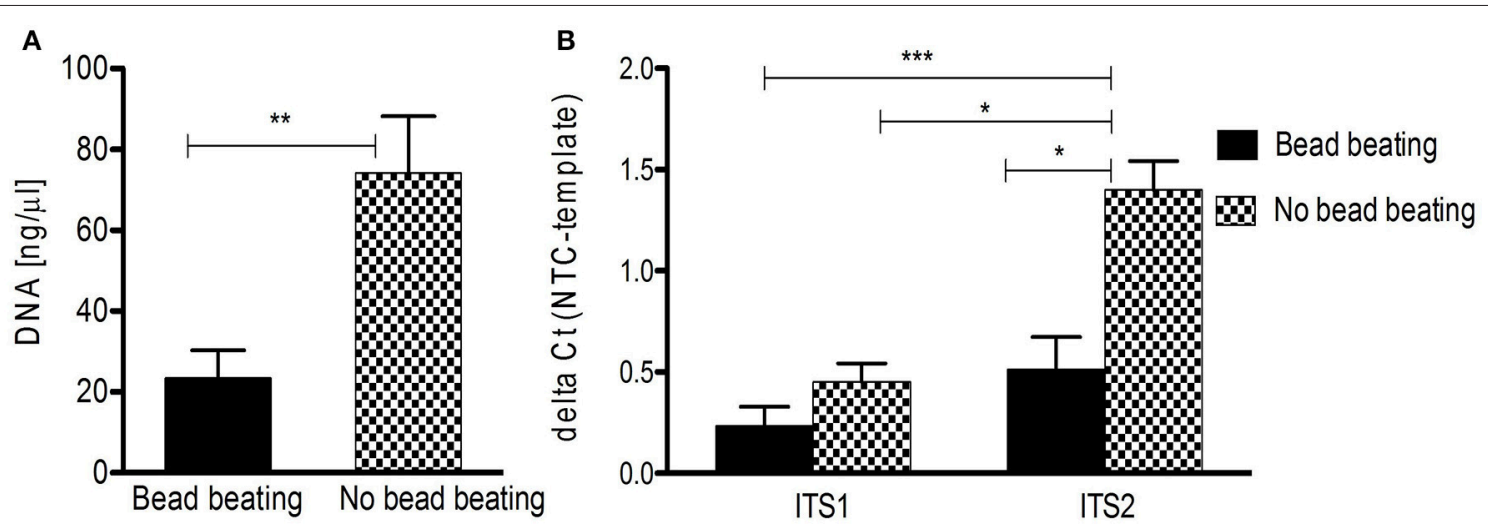

FIGURE 2 | DNA isolation from human FFPE skin samples and ITS PCR amplification influenced by beat beating. (A) Significant difference in overall DNA yield from FFPE skin samples $(n=10)$ with and without bead beating $\left({ }^{* *} p<0.005\right.$ by Mann Whitney test; data are mean + SEM). (B) Significantly increased detection of fungal DNA isolated without bead beating by ITS2 qPCR $\left(n=10 ;{ }^{*} p<0.05,{ }^{* \star \star} p<0.005\right.$, Kruskal-Wallis test; data are mean + SEM). NTC, negative control.

TABLE 1 | Overview of commonly used ITS1 and ITS2 oligonucleotide primer pairs.

\begin{tabular}{|c|c|c|c|c|c|c|c|}
\hline Region & Name & Sequence (Forward) & Name & Sequence (Reverse) & $\begin{array}{l}\text { Length } \\
\text { (bp) }\end{array}$ & $\begin{array}{l}\mathrm{Tm} \\
\left({ }^{\circ} \mathrm{C}\right)\end{array}$ & References \\
\hline \multirow[t]{3}{*}{ ITS1 } & ITS1 & TCCGTAGGTGAACCTGCGG & ITS2 & GCTGCGTTCTTCATCGATGC & $\sim 290$ & 65 & $\begin{array}{l}\text { White et al., 1990; } \\
\text { Muñoz-Cadavid et al., } \\
\text { 2010; Schoch et al., } 2012\end{array}$ \\
\hline & ITS5 & GGAAGTAAAAGTCGTAACAAGG & ITS2 & GCTGCGTTCTTCATCGATGC & $\sim 315$ & 63 & White et al., 1990 \\
\hline & ITS1F & СTTGGTCATTTAGAGGAAGTAA & ITS2 & GCTGCGTTCTTCATCGATGC & $\sim 350$ & 51 & Mello et al., 2011 \\
\hline \multirow[t]{2}{*}{ ITS2 } & ITS3 & GCATCGATGAAGAACGCAGC & ITS4 & TCCTCCGCTTATTGATATGC & $\sim 330$ & 62 & $\begin{array}{l}\text { White et al., 1990, } \\
\text { Muñoz-Cadavid et al., } \\
\text { 2010; Mello et al., 2011; } \\
\text { Flury et al., } 2014\end{array}$ \\
\hline & $5.8 S-F$ & GTGAATCATCGARTCTTTGAAC & $28 S 1-R$ & ATGCTTAAGTTCAGCGGGTA & $\sim 300$ & ${ }^{*} \mathrm{~ns}$ & Findley et al., 2013 \\
\hline \multirow[t]{3}{*}{$\begin{array}{l}\text { ITS1-2 incl. } \\
5.8 S \text { rRNA } \\
\text { gene }\end{array}$} & ITS5 & GGAAGTAAAAGTCGTAACAAGG & ITS4 & TCCTCCGCTTATTGATATGC & $\sim 641$ & 58 & $\begin{array}{l}\text { White et al., 1990; } \\
\text { Muñoz-Cadavid et al., } \\
\text { 2010; Flury et al., } 2014\end{array}$ \\
\hline & ITS1F & CTTGGTCATTTAGAGGAAGTAA & ITS4-B & CAGGAGACTTGTACACGGTCCAG & $\sim 600$ & 55 & Gardes and Bruns, 1993 \\
\hline & ITS1-F_KYO2 & TAGAGGAAGTAAAAGTCGTAA & ITS4 & TCCTCCGCTTATTGATATGC & $\sim 700$ & 47 & Toju et al., 2012 \\
\hline
\end{tabular}

*ns, not specified.

These difficulties make a thorough review of the (pre-) analytical process of mycobiota studies mandatory. To highlight the influence of pre-analytics on ITS based mycobiota investigations we assessed the performance of DNA extraction from human skin FFPE samples (see Supplementary Table S1 for sample information) with a commercially available kit (QIAamp 
DNA FFPE tissue kit, Qiagen) reported to be efficient for fungal DNA extraction out of FFPE material (Muñoz-Cadavid et al., 2010). We added a mechanical cell disruption step (bead-beating) to the procedure (MagnaLyser, Roche), since this step was shown to be crucial for complete lysis of microbial cells in specimens, significantly influencing correct community representation (de Boer et al., 2010; Reck et al., 2015). A detailed description of the applied method is given in the Data Sheet S1. Interestingly, we observed that bead-beating significantly lead to lower DNA yields and a significantly decreased signal-to-noise ratio in ITS PCR, impairing efficient fungal PCR amplification (Figure 2). Thus, mechanical lysis of specimens could also counteract reliable mycobiota investigations especially if low-biomass samples such as skin are used.

\section{ITS AMPLIFICATION VIA PCR}

For amplification of fungal DNA various primers have been designed targeting different regions of the rRNA operon or other marker genes encoding translation elongation factor 1$\alpha$, RNA polymerase II, $\beta$-tubulin, and the minichromosome maintenance complex component 7 (MCM7) protein (White et al., 1990; Tanabe et al., 2002; McLaughlin et al., 2009; O'Donnell et al., 2010; Schoch et al., 2012; Toju et al., 2012; Lindahl et al., 2013). Of these, the ITS regions are considered the formal barcode for fungal taxonomy (Schoch et al., 2012; Lindahl et al., 2013). As noted above, ITS1 and ITS2 sequences are highly variable and can be used to discriminate fungi even down to species level (Martin and Rygiewicz, 2005; Porras-Alfaro et al., 2014). However, each ITS primer combination fails to amplify certain species, a situation similar to bacterial 16S rRNA gene based analysis (Bellemain et al., 2010). Thus the use of multiple primer combinations and/or primers with degenerated nucleotide positions is recommended to capture the entire fungal community (Ihrmark et al., 2012; Toju et al., 2012). Table 1 summarizes commonly used ITS1 and ITS2 oligonucleotide primers. Of note, the ITS2 region was reported to perform better for fungal DNA amplification out of FFPE material (MuñozCadavid et al., 2010; Flury et al., 2014). We also observed increased PCR performance using ITS2 primers and human skin FFPE samples (Figure 2B). However, other reports obtained similar amplification rates with ITS1 and ITS2 oligonucleotides (Mello et al., 2011; Bazzicalupo et al., 2013; Blaalid et al., 2013; Lindahl et al., 2013).

\section{BIOINFORMATICS CHALLENGES IN MYCOBIOTA ANALYSES}

The bioinformatics analysis workflow of amplicon data can be summarized into four main steps: (i) pre-processing, (ii) OTU picking, (iii) taxonomic classification, and (iv) visualization and statistical analysis (Figure 3; Kuczynski et al., 2012). So far dedicated bioinformatics tools for mycobiota analyses are sparse. Measures originally developed for $16 \mathrm{~S}$ rRNA gene data, like QIIME (Caporaso et al., 2010) and mothur (Schloss et al., 2009) are often employed to investigate ITS amplicons. However, these

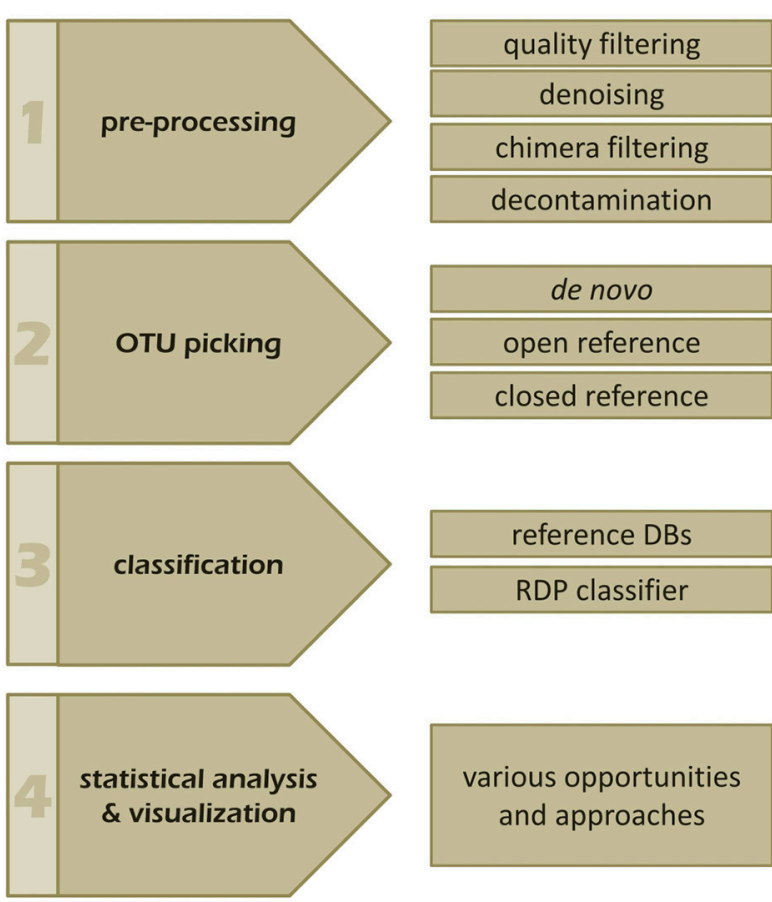

FIGURE 3 | The four main steps of a typical amplicon analysis workflow. Individual steps and features of (1) pre-processing, (2) OTU picking, (3) taxonomic annotation, as well as, (4) visualization and statistics are indicated and discussed in the manuscript.

tools pose several shortcomings when applied to ITS sequences, especially when standard protocols are used. In the following the main analytical steps and potential hurdles of ITS based amplicon data analyses are discussed with special emphasis on OTU clustering (OTU picking) and classification. We also highlight the effect of different OTU picking strategies on taxonomic classification of ITS data by comparative analysis of an ITS1 in silico mock community.

\section{PRE-PROCESSING OF AMPLICON RAW DATA}

Current pre-processing recommendations include rigorous length filtering of reads, noise reduction (detection, correction, and removal of sequencing errors and artifacts), quality filtering (removal of reads with quality scores below a defined threshold; average $>25$ ), chimera removal (detection and removal of artificially created reads, produced different targets during PCR), as well as removal of singletons/doubletons (Bokulich et al., 2013). The latter could emerge due to sequencing errors (e.g., within homopolymers) leading to OTU inflation of data, which is dependent also on the sequencing technology used (Schirmer et al., 2015). Choice of pre-processing methods and used parameters heavily influence the number of created OTUs, which could lead to underestimation of species diversity if too stringent filtering is applied (Flynn et al., 2015; Kopylova et al., 2016). However, adequate pre-processing of raw reads is mandatory 
TABLE 2 | List of commonly used clustering algorithms.

\begin{tabular}{|c|c|c|c|c|}
\hline Algorithm name & Algorithm type & Multiple sequence alignment required & Integrated in & References \\
\hline Mothur & Hierarchical & YES & Mothur & Schloss et al., 2009 \\
\hline UCLUST & Greedy & $\mathrm{NO}$ & QIIME & Edgar, 2010 \\
\hline UPARSE & Greedy & $\mathrm{NO}$ & QIIME & Caporaso et al., 2010; Edgar, 2013; Albanese et al., 2015 \\
\hline SWARM & Agglomerative & $\mathrm{NO}$ & QIIME, MICCA & Caporaso et al., 2010; Mahé et al., 2014; Albanese et al., 2015 \\
\hline OTUCLUST & Greedy & NO & MICCA & Albanese et al., 2015 \\
\hline
\end{tabular}

A

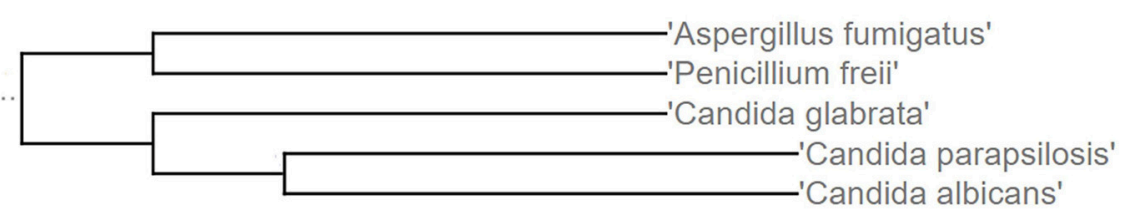

B

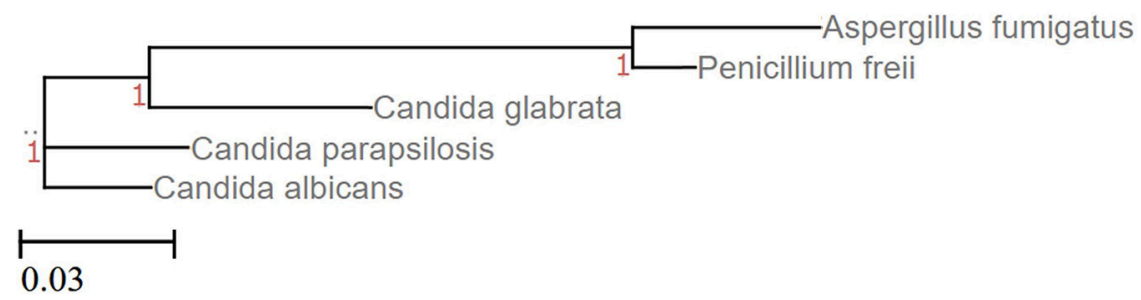

C

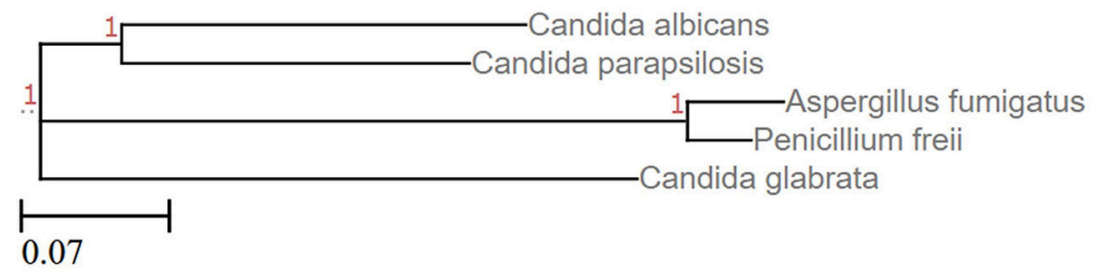

FIGURE 4 | Phylogenetic resolution of five different fungal species is impaired when clustering ITS sequences. (A) Tree based on the corresponding NCBI taxonomy information using NCBl's Common Tree. Treeing is congruent with the phylogenetic study performed by Diezmann et al. (2004). (B) LSU based treeing recapitulates largely the NCBI taxonomy. (C) ITS based treeing impairs phylogeny. Trees of subfigures (B,C) are based on MSA of LSU and ITS2 fragments,

respectively (taxon IDs and accession numbers are given as Data Sheets S3-S5).

independent of the used maker gene, leading to a reduced number of assigned OTUs and less noise in the data. Basically we refer to the suggestions of Schloss et al. (2011), but as there are no general rules for pre-processing we strongly recommend looking carefully into what is happening during filtering rather than just applying default parameters.

\section{OTU PICKING-CLUSTERING INTO OPERATIONAL TAXONOMIC UNITS (OTUS)}

Numerous approaches and tools are available for clustering sequences into OTUs. Current algorithms developed primarily for 16S rRNA gene amplicons are summarized in Table 2. In general OTU clustering and annotation could be achieved by using three different strategies (i) de novo-, (ii) closed reference-, and (iii) open reference-based clustering. Briefly, a closed reference approach calculates for each input sequence the best pairwise alignment to a pre-defined reference database collection. Sequences with the same best match are binned into the same cluster (i.e., OTU). In contrast, de novo based strategies cluster sequences within a pre-defined distance (commonly $3 \%$ ). For each of these clusters a representative sequence is selected and taxonomically classified. Open-reference OTU picking is a mixture of both. Reads are first clustered using a closed reference approach and all reads which fail in this first step are subsequently clustered using a de novo strategy (Rideout et al., 2014; Westcott and Schloss, 2015). A recent comparison of the three different clustering strategies revealed the de novo approach based on a global distance matrix (implemented by default by mothur) as the optimal method for clustering $16 \mathrm{~S}$ rRNA gene sequences into OTUs (Westcott and Schloss, 


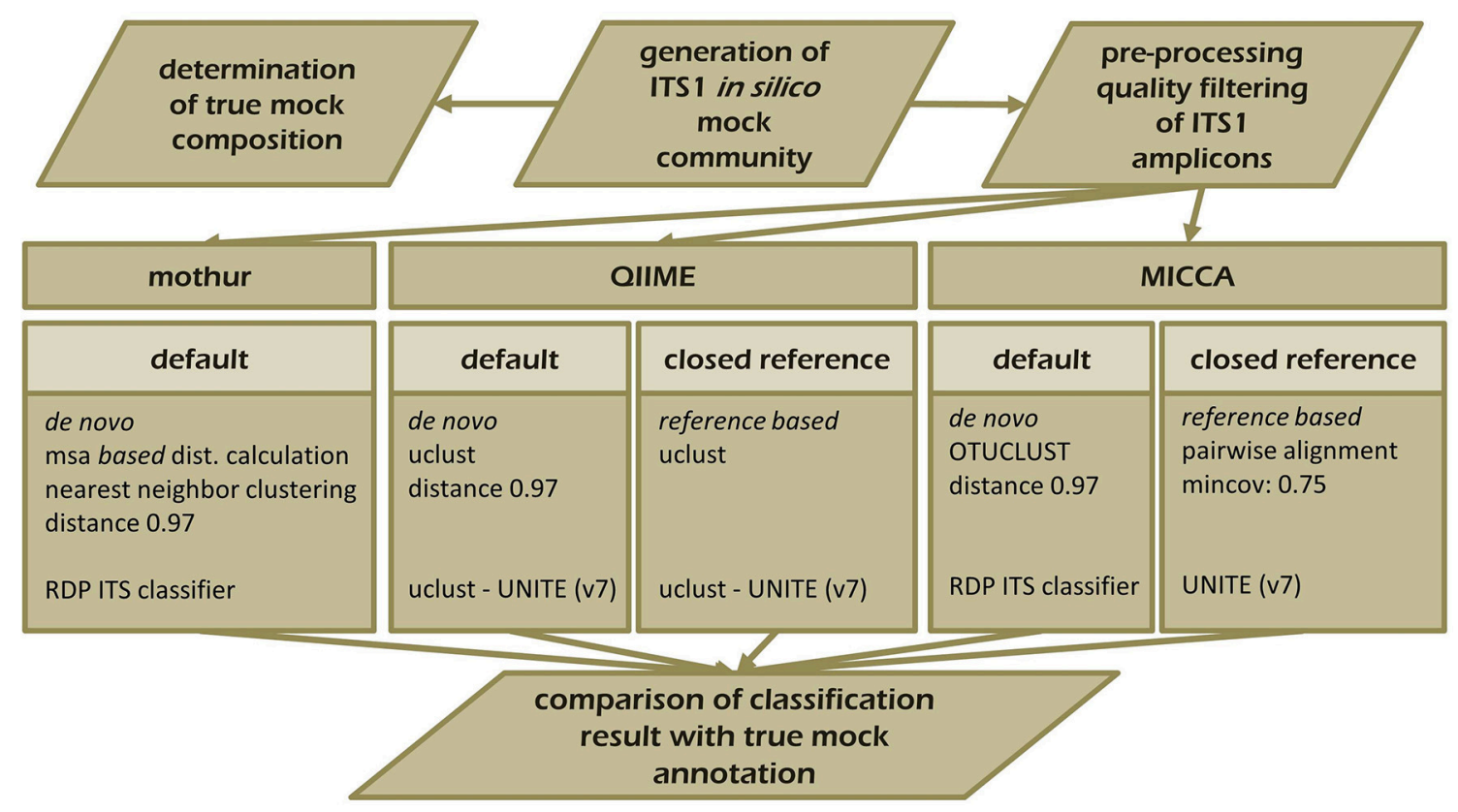

FIGURE 5 | Schematic overview of the experimental set-up testing the performance of mothur, QIIME, and MICCA to resolve the ITS1 mock community. ITS1 fragments were extracted from the UNITE ITS reference collection (v.7) and analyzed with mothur (default workflow), QIIME, and MICCA (default and closed reference based workflow).

2015). Such benchmark comparisons are unfortunately missing for ITS amplicons. Importantly, the use of multiple sequence alignments (MSA) for clustering ITS sequences in a de novo approach poses a significant problem. ITS sequences show a high degree of intraspecific variation (Figures 1D,E), which leads to the introduction of gaps during the alignment process and subsequently to erroneous multiple sequence alignments exhibiting wrong phylogenetic resolution (Figure 4). In addition, there is no commonly accepted genus or species level cutoff for the formation of ITS clusters, such as 5\% variation for genus- and 3\% for species-level clustering applied to $16 \mathrm{~S}$ rRNA gene data (Stackebrandt and Goebel, 1994). Often 3\% variation is used and this cut-off seems to perform reasonable for fungal ITS sequences, although taxonomic resolution is clearly impaired within certain taxa. Both, ITS1 and ITS2, show a highly congruent fungal taxonomic resolution (Blaalid et al., 2013).

\section{TAXONOMIC CLASSIFICATION OF OTUs}

If a closed reference-based approach is used, taxonomic classification is achieved already during the OTU picking step, wherein OTUs represent clusters of identical matches to the reference database. If a de novo strategy is employed a proxy sequence from each cluster is chosen and taxonomically classified either by calculating sequence similarities between the proxy sequence and a reference database or by estimating the classification confidence using a pre-trained classifier, such as the RDP classifier (Wang et al., 2007). The latter one offers training sets for ITS (Porras-Alfaro et al., 2014) as well as for LSU (Liu et al., 2012) sequences. Accurate taxonomic classification of sequences requires reference databases of high quality. The UNITE (Unified system for the DNA based fungal species linked to the classification, https://unite.ut.ee) database for ITS fragments represents a curated full-length ITS sequence repository devoid of ambiguous sequences (Nilsson et al., 2014). Several factors lead to misannotated ITS sequences in repositories, such as GenBank, EMBL, or DDJB. For instance many fungi have sexual (teleomorph) and asexual (anamorph) forms and they are often classified as different taxa assigned even to different families (Mahé et al., 2012; Underhill and Iliev, 2014). UNITE represents currently the most comprehensive taxonomic ITS classification resource, providing ready-to-use application files for mothur, QIIME, and MICCA. Although still some fungal lineages are uncovered it comprises 536,881 sequence entries (as of January 2016, UNITE version 7.0). Recently, the hand curated ISHAM-ITS reference DNA barcoding database, with 3,200 sequences covering about 415 fungal species (as of December 2015) maintained by the Society for Human and Animal Mycology (ISHAM) was incorporated into UNITE (Irinyi et al., 2015). Noteworthy is UNITE's key concept, the so-called species hypotheses $(\mathrm{SH})$. A SH represents an operational taxonomic unit at approximately species level (Kõljalg et al., 2013). Each SH is represented by the most homologous high quality sequence within a respective sequence cluster linked to a unique, permanent digital object identifier (DOI), which allows for unambiguous identification even in absence of a full formal taxonomic name or when a fungal OTU remains taxonomically unassigned. Of note, the global fungome is estimated to comprise 1.5-6 million different species (Hawksworth, 1991; Blackwell, 2011; Taylor et al., 
TABLE 3 | Correct classification of the in silico ITS1 mock community with different analysis pipelines and OTU picking strategies (\% in parenthesis).

\begin{tabular}{|c|c|c|c|c|c|c|c|c|}
\hline & \multirow{2}{*}{$\begin{array}{l}\text { No. of classified } \\
\text { sequences }\end{array}$} & \multirow[t]{2}{*}{ No. of OTUs } & \multicolumn{6}{|c|}{ Taxonomic classification } \\
\hline & & & Phylum & Class & Order & Family & Genus & Species \\
\hline In silico ITS1 mock & 56,451 & 11,336 & 6 & 45 & 143 & 409 & 1,931 & 11,336 \\
\hline Mothur & 36,255 & 26,965 & 35,675 (98.40) & 33,686 (92.91) & 32,949 (90.88) & 29,739 (82.02) & $24,000(66.20)$ & $11,233(30.98)$ \\
\hline QIIME default & 56,451 & 19,779 & $18,930(33.53)$ & $14,316(25.36)$ & 4,865 (8.62) & $669(1.18)$ & $372(0.65)$ & $37(0.07)$ \\
\hline MICCA closed reference & 52,475 & 9,942 & 52,129 (99.34) & $49,670(94.65)$ & 49,434 (94.20) & $45,784(87.25)$ & $41,206(78.52)$ & $26,400(50.31)$ \\
\hline
\end{tabular}

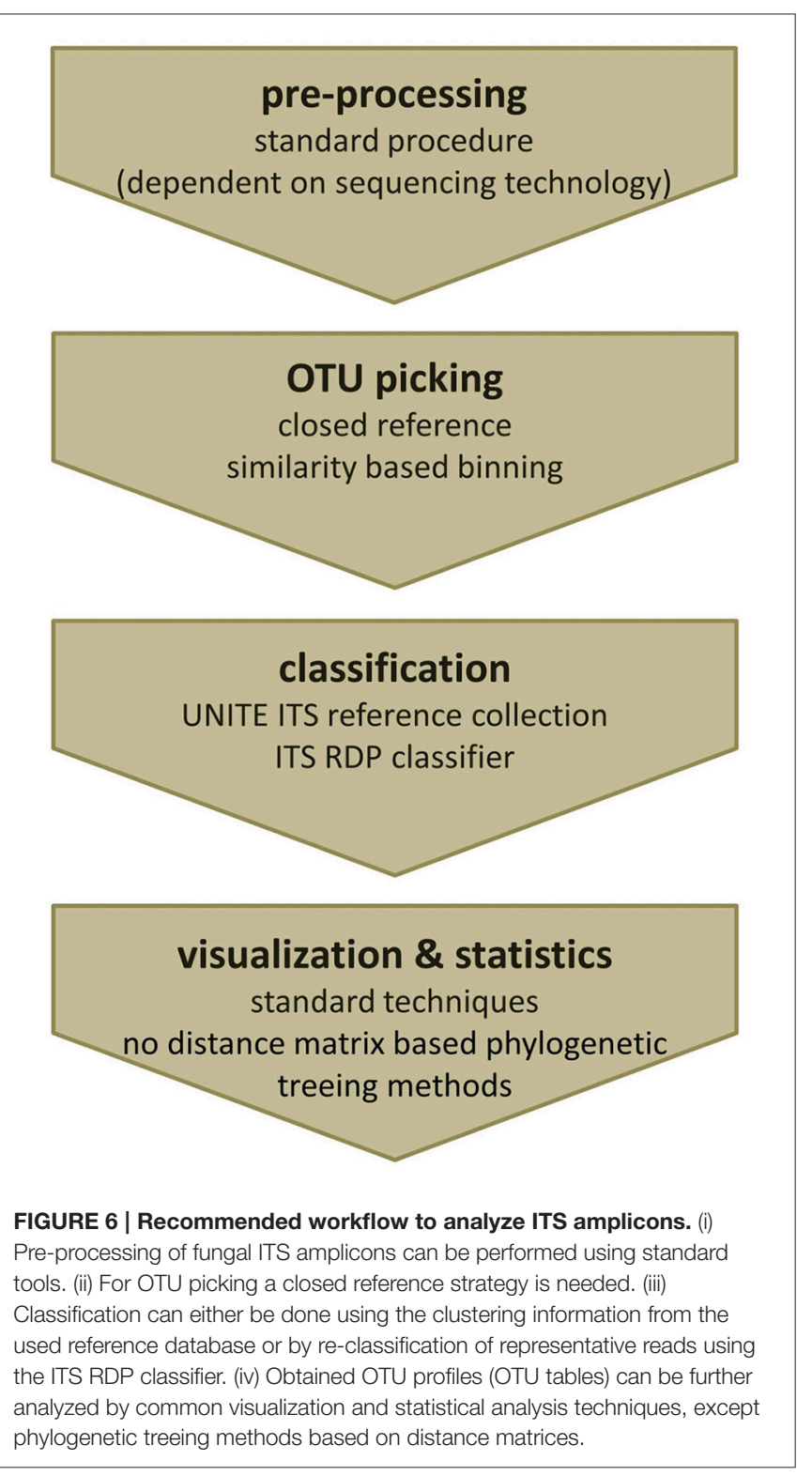

2014), wherein currently 130,000 species are represented in the public sequence repositories (http://www.speciesfungorum.org/, accessed March 2016). These counts give already an idea about the "completeness" of the current fungal reference databases (Tedersoo et al., 2014).

\section{THE EFFECT OF DIFFERENT OTU PICKING STRATEGIES ON TAXONOMIC CLASSIFICATION OF ITS DATA}

To demonstrate the influence of different OTU picking strategies on phylogenetic resolution of fungal communities we compared three commonly used analysis pipelines mothur, QIIME, and MICCA, employing an in silico created fungal ITS1 mock community. Therefore, 582,779 ITS1 fragments were extracted by ITSx (Bengtsson-Palme et al., 2013) from the public UNITE sequence collection (version 7, comprising 656,899 sequences). Amplicons were filtered for ambiguous lineage definitions, resulting in 345,201 sequences. These amplicons were quality filtered yielding finally 56,451 unique ITS1 fragments (accession numbers and taxonomic annotations are given in Supplementary Table S2). ITS1 fragments were subsequently clustered into OTUs by the default de novo strategies employed by mothur, QIIME, and MICCA, according to the standard protocol of each pipeline (for details see Data Sheet S1). For analyses with QIIME and MICCA, sequences were additionally binned into OTUs according to their taxonomic classification using a closed reference OTU picking strategy employing the UNITE database (version 7, 22.08.2016). The database was used for classification of representative sequences either directly for similarity-based comparisons or indirectly for training the RDP classifier. Finally, the assigned taxonomic classifications were compared to the true annotation of the ITS1 mock community. A scheme highlighting the experimental design and used parameters for comparison of pipelines is shown in Figure 5. Table 3 summarizes the comparison results, which clearly indicates that choice of the OTU picking strategy severely impacts the phylogenetic resolution of the ITS mock community. All pipelines used with default parameters failed to accurately classify the mock community down to species level. All approaches classified ITS1 reads with a reasonable accuracy only to the order level (range 87.61-97.34\% correct assignment), except QIIME with default settings (de novo), which behaved poor (classifying only $33.53 \%$ of sequences correctly at phylum level and $0.07 \%$ at species level). A high number of singletons emerged by using all three de novo 
approaches, leading to OTU inflation, and wrongly clustered OTUs. Importantly, changing the default OTU picking approach of QIIME (de novo) to a closed reference approach increased the amount of correctly classified species to $71.62 \%$ (Table 3 ). Taken together these data indicate that closed reference based strategies should be preferred if ITS amplicons are analyzed. Nevertheless, a relatively large fraction of wrongly annotated OTUs might still persist, thus manual correction of taxonomic assignments (i.e., by individual blast analysis of sequences) might still improve classification (Iliev et al., 2012).

\section{VISUALIZATION AND STATISTICAL ANALYSIS OF ITS DATA}

Visualization and statistical analyses of mycobiota data typically enable measures for community structure, such as alpha-diversity metrics (e.g., richness, evenness, Shannon index), as well as taxonomic turnover (i.e., changes in microbial composition between conditions or groups) called beta-diversity, which can be calculated with different distance measurements (Bray Curtis, Andernberg, UniFrac, etc.). Principle coordinates analysis (PCoA) plots based on these distance matrices enable simplified visualization of the structural resemblance of mycobiota profiles. Statistical identification of differential abundant taxa between groups could be achieved using tools such as LEfSe (Segata et al., 2011) or linear modeling approaches, such as DESeq (Paulino et al., 2006) or edgeR (Robinson et al., 2010). Measures for alphaand beta-diversity are readily provided by tools such as mothur and QIIME and operate on the created OTU tables. Caution must be taken if measures derive phylogenetic information based on diversity matrices emerging from MSAs of ITS reads, such as UniFrac (Lozupone et al., 2011). Such methods lead to erroneous results because of the bad performance of aligning ITS reads as shown above (Figure 4).

\section{CONCLUSION}

Fungal amplicon studies benefit greatly from the advancements made in the analysis of bacterial communities, nonetheless, many hurdles need still to be solved and standards are waiting to be defined. Although numerous protocols and kits are available for fungal DNA isolation out of complex specimens such as human tissue, protocols need to be adapted to the special study needs. Recommendations on how to perform ITS analyses using mothur and QIIME with non-phylogenetic diversity metrics have been recently released (e.g., https://mothur.org/wiki/ Analysis_examples\#Sanger_16S-ITS_rRNA_sequence_analysis,

\section{REFERENCES}

Albanese, D., Fontana, P., De Filippo, C., Cavalieri, D., and Donati, C. (2015). MICCA: a complete and accurate software for taxonomic profiling of metagenomic data. Sci. Rep. 5:9743. doi: 10.1038/srep09743

Bazzicalupo, A. L., Bálint, M., and Schmitt, I. (2013). Comparison of ITS1 and ITS2 rDNA in 454 sequencing of hyperdiverse fungal communities. Fungal Ecol. 6, 102-109. doi: 10.1016/j.funeco.2012.09.003 accessed February 2017, http://qiime.org/1.7.0/tutorials/fungal_ its_analysis.html, accessed April 2016). Based on our experience, pre-processing, and quality filtering of ITS sequencing data, as well as chimera filtering could be done with standard 16S rRNA gene based procedures. We use the default workflow of mothur for ITS data pre-processing, assembling of paired reads, length-, quality-, and chimera filtering, as well as noise reduction as described in the MiSeq 16S SOP of Kuczynski et al. (2012, accessed May 2016). Since mothur employs pairwise distance matrices, which require the creation of multiple sequence alignments, we recommend switching to tools such as QIIME or MIICA for further analyses, which allow for closed reference-based approaches. Subsequently QIIME can be used for visualization of mycobiota data. The crucial step within QIIME is to suppress tree generation within the OTU picking step and to use closed reference OTU picking instead of the default de novo strategy. The pre-formatted version of the UNITE ITS reference database which is provided directly by UNITE works perfectly with one of the reference-based OTU picking scripts of QIIME and MICCA. Alternatively sequences can be also classified and binned based on the information gained by the RDP classifier trained for ITS fragments or simply by an individual blast approach. A final summary of the recommended analysis steps for ITS based mycobiota analysis is given in Figure 6.

\section{AUTHOR CONTRIBUTIONS}

Conceptualization: BH, RN, GT, and GG. Data analysis: BH and NM. Manuscript draft: $\mathrm{BH}$ and GG. Final manuscript and approval: All authors.

\section{FUNDING}

This work was supported by BioTechMed-Graz and the Austrian Science Fund (FWF W1241-B18).

\section{ACKNOWLEDGMENTS}

Jürgen C. Becker, Karl Kashofer, and Andrea Thüringer are acknowledged for their input regarding mycobiota analysis.

\section{SUPPLEMENTARY MATERIAL}

The Supplementary Material for this article can be found online at: http://journal.frontiersin.org/article/10.3389/fmicb. 2017.00180/full\#supplementary-material 
ITS2 from ribosomal ITS sequences of fungi and other eukaryotes for analysis of environmental sequencing data. Methods Ecol. Evol. 4, 914-919. doi: 10.1111/2041-210x.12073

Blaalid, R., Kumar, S., Nilsson, R. H., Abarenkov, K., Kirk, P. M., and Kauserud, H. (2013). ITS1 versus ITS2 as DNA metabarcodes for fungi. Mol. Ecol. Resour. 13, 218-224. doi: 10.1111/1755-0998.12065

Blackwell, M. (2011). The fungi: 1, 2, 3... 5.1 million species? Am. J. Bot. 98, 426-438. doi: 10.3732/ajb.1000298

Bokulich, N. A., Subramanian, S., Faith, J. J., Gevers, D., Gordon, J. I., Knight, R., et al. (2013). Quality-filtering vastly improves diversity estimates from Illumina amplicon sequencing. Nat. Methods 10, 57-59. doi: 10.1038/nmeth.2276

Bonin, S., and Stanta, G. (2013). Nucleic acid extraction methods from fixed and paraffin-embedded tissues in cancer diagnostics. Expert Rev. Mol. Diagn. 13, 271-282. doi: 10.1586/erm.13.14

Brown, G. D., Denning, D. W., Gow, N. A., Levitz, S. M., Netea, M. G., and White, T. C. (2012). Hidden killers: human fungal infections. Sci. Transl. Med. 4:165rv13. doi: 10.1126/scitranslmed.3004404

Caporaso, J. G., Kuczynski, J., Stombaugh, J., Bittinger, K., Bushman, F. D., Costello, E. K., et al. (2010). QIIME allows analysis of highthroughput community sequencing data. Nat. Methods 7, 335-336. doi: $10.1038 /$ nmeth.f.303

Charlson, E. S., Diamond, J. M., Bittinger, K., Fitzgerald, A. S., Yadav, A., Haas, A. R., et al. (2012). Lung-enriched organisms and aberrant bacterial and fungal respiratory microbiota after lung transplant. Am. J. Respir. Crit. Care Med. 186, 536-545. doi: 10.1164/rccm.201204-0693OC

Coura, R., Prolla, J. C., Meurer, L., and Ashton-Prolla, P. (2005). An alternative protocol for DNA extraction from formalin fixed and paraffin wax embedded tissue. J. Clin. Pathol. 58, 894-895. doi: 10.1136/jcp.2004.021352

Cui, L., Morris, A., and Ghedin, E. (2013). The human mycobiome in health and disease. Genome Med. 5:63. doi: 10.1186/gm467

de Boer, R., Peters, R., Gierveld, S., Schuurman, T., Kooistra-Smid, M., and Savelkoul, P. (2010). Improved detection of microbial DNA after bead-beating before DNA isolation. J. Microbiol. Methods 80, 209-211. doi: 10.1016/j.mimet.2009.11.009

Diezmann, S., Cox, C. J., Schonian, G., Vilgalys, R. J., and Mitchell, T. G. (2004). Phylogeny and evolution of medical species of Candida and related taxa: a multigenic analysis. J. Clin. Microbiol. 42, 5624-5635. doi: 10.1128/JCM.42.12.5624-5635.2004

Edgar, R. C. (2010). Search and clustering orders of magnitude faster than BLAST. Bioinformatics 26, 2460-2461. doi: 10.1093/bioinformatics/btq461

Edgar, R. C. (2013). UPARSE: highly accurate OTU sequences from microbial amplicon reads. Nat. Methods 10, 996-998. doi: 10.1038/nmeth.2604

Findley, K., Oh, J., Yang, J., Conlan, S., Deming, C., Meyer, J. A., et al. (2013). Topographic diversity of fungal and bacterial communities in human skin. Nature 498, 367-370. doi: 10.1038/nature12171

Flury, B., Weisser, M., Prince, S. S., Bubendorf, L., Battegay, M., Frei, R., et al. (2014). Performances of two different panfungal PCRs to detect mould DNA in formalin-fixed paraffin-embedded tissue: what are the limiting factors? BMC Infect. Dis. 14:692. doi: 10.1186/s12879-014-0692-Z

Flynn, J. M., Brown, E. A., Chain, F. J., MacIsaac, H. J., and Cristescu, M. E. (2015). Toward accurate molecular identification of species in complex environmental samples: testing the performance of sequence filtering and clustering methods. Ecol. Evol. 5, 2252-2266. doi: 10.1002/ece3.1497

Gardes, M., and Bruns, T. D. (1993). ITS primers with enhanced specificity for basidiomycetes - application to the identification of mycorrhizae and rusts. Mol. Ecol. 2, 113-118. doi: 10.1111/j.1365-294X.1993.tb00005.x

Ghannoum, M. A., Jurevic, R. J., Mukherjee, P. K., Cui, F., Sikaroodi, M., Naqvi, A., et al. (2010). Characterization of the oral fungal microbiome (mycobiome) in healthy individuals. PLoS Pathog. 6:e1000713. doi: 10.1371/journal.ppat.1000713

Goldschmidt, P., Degorge, S., Merabet, L., and Chaumeil, C. (2014). Enzymatic treatment of specimens before DNA extraction directly influences molecular detection of infectious agents. PLOS ONE 9:e94886. doi: 10.1371/journal.pone.0094886

Gorkiewicz, G., Thallinger, G. G., Trajanoski, S., Lackner, S., Stocker, G., Hinterleitner, T., et al. (2013). Alterations in the colonic microbiota in response to osmotic diarrhea. PLoS ONE 8:e55817. doi: 10.1371/journal.pone.0055817
Gosiewski, T., Salamon, D., Szopa, M., Sroka, A., Malecki, M. T., and Bulanda, M. (2014). Quantitative evaluation of fungi of the genus Candida in the feces of adult patients with type 1 and 2 diabetes - a pilot study. Gut Pathog. 6:43. doi: 10.1186/s13099-014-0043-z

Gouba, N., and Drancourt, M. (2015). Digestive tract mycobiota: a source of infection. Med. Mal. Infect. 45, 9-16. doi: 10.1016/j.medmal.2015. 01.007

Hallen-Adams, H. E., Kachman, S. D., Kim, J., Legge, R. M., and Martínez, I. (2015). Fungi inhabiting the healthy human gastrointestinal tract: a diverse and dynamic community. Fungal Ecol. 15, 9-17. doi: 10.1016/j.funeco.2015. 01.006

Hartmann, M., Howes, C. G., Abarenkov, K., Mohn, W. W., and Nilsson, R. H. (2010). V-Xtractor: an open-source, high-throughput software tool to identify and extract hypervariable regions of small subunit (16S/18S) ribosomal RNA gene sequences. J. Microbiol. Methods 83, 250-253. doi: 10.1016/j.mimet.2010.08.008

Hawksworth, D. (1991). The fungal dimension of biodiversity: magnitude, significance, and conservation. Mycol. Res. 95, 641-655. doi: 10.1016/S0953-7562(09)80810-1

Hoarau, G., Mukherjee, P. K., Gower-Rousseau, C., Hager, C., Chandra, J., Retuerto, M. A., et al. (2016). Bacteriome and mycobiome interactions underscore microbial dysbiosis in familial Crohn's disease. MBio 7, e01250e01216. doi: 10.1128/mBio.01250-16

Hunt, J., Boddy, L., Randerson, P. F., and Rogers, H. J. (2004). An evaluation of $18 \mathrm{~S}$ rDNA approaches for the study of fungal diversity in grassland soils. Microb. Ecol. 47, 385-395. doi: 10.1007/s00248-003-2018-3

Ihrmark, K., Bödeker, I. T., Cruz-Martinez, K., Friberg, H., Kubartova, A., Schenck, J., et al. (2012). New primers to amplify the fungal ITS2 region - evaluation by 454-sequencing of artificial and natural communities. FEMS Microbiol. Ecol. 82, 666-677. doi: 10.1111/j.1574-6941.2012.01437.x

Iliev, I. D., Funari, V. A., Taylor, K. D., Nguyen, Q., Reyes, C. N., Strom, S. P., et al. (2012). Interactions between commensal fungi and the Ctype lectin receptor Dectin-1 influence colitis. Science 336, 1314-1317. doi: $10.1126 /$ science. 1221789

Irinyi, L., Serena, C., Garcia-Hermoso, D., Arabatzis, M., Desnos-Ollivier, M., $\mathrm{Vu}, \mathrm{D}$., et al. (2015). International Society of Human and Animal Mycology (ISHAM)-ITS reference DNA barcoding database - the quality controlled standard tool for routine identification of human and animal pathogenic fungi. Med. Mycol. 53, 313-337. doi: 10.1093/mmy/myv008

Kappe, R., Fauser, C., Okeke, C. N., and Maiwald, M. (1996). Universal fungusspecific primer systems and group-specific hybridization oligonucleotides for 18S rDNA. Mycoses 39, 25-30. doi: 10.1111/j.1439-0507.1996.tb00079.x

Kim, S. H., Clark, S. T., Surendra, A., Copeland, J. K., Wang, P. W., Ammar, R., et al. (2015). Global analysis of the fungal microbiome in cystic fibrosis patients reveals loss of function of the transcriptional repressor nrg1 as a mechanism of pathogen adaptation. PLoS Pathog. 11:e1005308. doi: 10.1371/journal.ppat.1005308

Kocjan, B. J., Hosnjak, L., and Poljak, M. (2015). Commercially available kits for manual and automatic extraction of nucleic acids from formalin-fixed, paraffinembedded (FFPE) tissues. Acta Dermatovenerol. Alp. Pannonica. Adriat. 24, 47-53. doi: 10.15570/actaapa.2015.12

Kõljalg, U., Nilsson, R. H., Abarenkov, K., Tedersoo, L., Taylor, A. F. S. Bahram, M., et al. (2013). Towards a unified paradigm for sequencebased identification of fungi. Mol. Ecol. 22, 5271-5277. doi: 10.1111/mec. 12481

Kopylova, E., Navas-Molina, J. A., Mercier, C. Ã., Xu, Z. Z., Mahé, F., He, Y., et al. (2016). Open-source sequence clustering methods improve the state of the art. mSystems 1, e00003-e00015. doi: 10.1128/mSystems.00003-15

Krause, R., Halwachs, B., Thallinger, G. G., Klymiuk, I., Gorkiewics, G., Hoenigl, M., et al. (2016). Characterisation of Candida within the mycobiome/microbiome of the lower respiratory tract of ICU patients. PLoS ONE 11:e0155033. doi: 10.1371/journal.pone.0155033

Kuczynski, J., Lauber, C. L., Walters, W. A., Parfrey, L. W., Clemente, J. C., Gevers, D., et al. (2012). Experimental and analytical tools for studying the human microbiome. Nat. Rev. Genet. 13, 47-58. doi: 10.1038/nrg3129

Lindahl, B. D., Nilsson, R. H., Tedersoo, L., Abarenkov, K., Carlsen, T., Kjoller, R., et al. (2013). Fungal community analysis by high-throughput 
sequencing of amplified markers - a user's guide. New Phytol. 199, 288-299. doi: $10.1111 /$ nph. 12243

Liu, K. L., Porras-Alfaro, A., Kuske, C. R., Eichorst, S. A., and Xie, G. (2012). Accurate, rapid taxonomic classification of fungal large-subunit rRNA genes. Appl. Environ. Microbiol. 78, 1523-1533. doi: 10.1128/AEM.06826-11

Lozupone, C., Lladser, M. E., Knights, D., Stombaugh, J., and Knight, R. (2011). UniFrac: an effective distance metric for microbial community comparison. ISME J. 5, 169-172. doi: 10.1038/ismej.2010.133

Mahé, F., Rognes, T., Quince, C., de Vargas, C., and Dunthorn, M. (2014). Swarm: robust and fast clustering method for amplicon-based studies. PeerJ 2:e593. doi: $10.7717 /$ peerj.593

Mahé, S., Duhamel, M., Le Calvez, T., Guillot, L., Sarbu, L., Bretaudeau, A., et al. (2012). PHYMYCO-DB: a curated database for analyses of fungal diversity and evolution. PLOS ONE 7:e43117. doi: 10.1371/journal.pone. 0043117

Martin, K. J., and Rygiewicz, P. T. (2005). Fungal-specific PCR primers developed for analysis of the ITS region of environmental DNA extracts. BMC Microbiol. 5:28. doi: 10.1186/1471-2180-5-28

McLaughlin, D. J., Hibbett, D. S., Lutzoni, F., Spatafora, J. W., and Vilgalys, R. (2009). The search for the fungal tree of life. Trends Microbiol. 17, 488-497. doi: 10.1016/j.tim.2009.08.001

Mello, A., Napoli, C., Murat, C., Morin, E., Marceddu, G., and Bonfante, P. (2011). ITS-1 versus ITS-2 pyrosequencing: a comparison of fungal populations in truffle grounds. Mycologia 103, 1184-1193. doi: 10.3852/11-027

Muñoz-Cadavid, C., Rudd, S., Zaki, S. R., Patel, M., Moser, S. A., Brandt, M. E., et al. (2010). Improving molecular detection of fungal DNA in formalin-fixed paraffin-embedded tissues: comparison of five tissue DNA extraction methods using panfungal PCR. J. Clin. Microbiol. 48, 2147-2153. doi: 10.1128/JCM.00459-10

Nilsson, R. H., Hyde, K., Pawlowska, J., Ryberg, M., Tedersoo, L., Aas, A. B., et al. (2014). Improving ITS sequence data for identification of plant pathogenic fungi. Fungal Divers. 67, 11-19. doi: 10.1007/s13225-014-0291-8

O’Donnell, K., Sutton, D. A., Rinaldi, M. G., Sarver, B. A., Balajee, S. A., Schroers, H. J., et al. (2010). Internet-accessible DNA sequence database for identifying fusaria from human and animal infections. J. Clin. Microbiol. 48, 3708-3718. doi: 10.1128/JCM.00989-10

Oever, J. T., and Netea, M. G. (2014). The bacteriome-mycobiome interaction and antifungal host defense. Eur. J. Immunol. 44, 3182-3191. doi: $10.1002 /$ eji.201344405

Oh, J., Byrd, A. L., Deming, C., Conlan, S., Kong, H. H., and Segre, J. A. (2014). Biogeography and individuality shape function in the human skin metagenome. Nature 514, 59-64. doi: 10.1038/nature13786

Oh, J., Freeman, A. F., Park, M., Sokolic, R., Candotti, F., Holland, S. M., et al. (2013). The altered landscape of the human skin microbiome in patients with primary immunodeficiencies. Genome Res. 23, 2103-2114. doi: 10.1101 /gr.159467.113

Ott, S. J., Kühbacher, T., Musfeldt, M., Rosenstiel, P., Hellmig, S., Rehman, A., et al. (2008). Fungi and inflammatory bowel diseases: alterations of composition and diversity. Scand. J. Gastroenterol. 43, 831-841. doi: $10.1080 / 00365520801935434$

Paulino, L. C., Tseng, C. H., Strober, B. E., and Blaser, M. J. (2006). Molecular analysis of fungal microbiota in samples from healthy human skin and psoriatic lesions. J. Clin. Microbiol. 44, 2933-2941. doi: 10.1128/JCM. 00785-06

Porras-Alfaro, A., Liu, K. L., Kuske, C. R., and Xie, G. (2014). From genus to phylum: large-subunit and internal transcribed spacer rRNA operon regions show similar classification accuracies influenced by database composition. Appl. Environ. Microbiol. 80, 829-840. doi: 10.1128/AEM.02894-13

Reck, M., Tomasch, J., Deng, Z., Jarek, M., Husemann, P., and Wagner-Dobler, I. (2015). Stool metatranscriptomics: a technical guideline for mRNA stabilisation and isolation. BMC Genomics 16:494. doi: 10.1186/s12864-015-1694-y

Rideout, J. R., He, Y., Navas-Molina, J. A., Walters, W. A., Ursell, L. K., Gibbons, S. M., et al. (2014). Subsampled open-reference clustering creates consistent, comprehensive OTU definitions and scales to billions of sequences. PeerJ 2:e545. doi: 10.7717/peerj.545

Robinson, M. D., McCarthy, D. J., and Smyth, G. K. (2010). edgeR: a Bioconductor package for differential expression analysis of digital gene expression data. Bioinformatics 26, 139-140. doi: 10.1093/bioinformatics/btp616
Sangoi, A. R., Rogers, W. M., Longacre, T. A., Montoya, J. G., Baron, E. J., and Banaei, N. (2009). Challenges and pitfalls of morphologic identification of fungal infections in histologic and cytologic specimens: a ten-year retrospective review at a single institution. Am. J. Clin. Pathol. 131, 364-375. doi: 10.1309/AJCP99OOOZSNISCZ

Schirmer, M., Ijaz, U. Z., D’Amore, R., Hall, N., Sloan, W. T., and Quince, C. (2015). Insight into biases and sequencing errors for amplicon sequencing with the Illumina MiSeq platform. Nucleic Acids Res. 43:e37. doi: 10.1093/nar/ gku1341

Schloss, P. D., Gevers, D., and Westcott, S. L. (2011). Reducing the effects of PCR amplification and sequencing artifacts on 16S rRNA-based studies. PLoS ONE 6:e27310. doi: 10.1371/journal.pone.0027310

Schloss, P. D., Westcott, S. L., Ryabin, T., Hall, J. R., Hartmann, M., Hollister, E. B., et al. (2009). Introducing mothur: open-source, platformindependent, community-supported software for describing and comparing microbial communities. Appl. Environ. Microbiol. 75, 7537-7541. doi: 10.1128/AEM.01541-09

Schoch, C. L., Seifert, K. A., Huhndorf, S., Robert, V., Spouge, J. L., Levesque, C. A., et al. (2012). Nuclear ribosomal internal transcribed spacer (ITS) region as a universal DNA barcode marker for Fungi. Proc. Natl. Acad. Sci. U.S.A. 109, 6241-6246. doi: 10.1073/pnas.1117018109

Segata, N., Izard, J., Waldron, L., Gevers, D., Miropolsky, L., Garrett, W. S., et al. (2011). Metagenomic biomarker discovery and explanation. Genome Biol. 12:R60. doi: 10.1186/gb-2011-12-6-r60

Simon, L., Lalonde, M., and Bruns, T. D. (1992). Specific amplification of $18 \mathrm{~S}$ fungal ribosomal genes from vesicular-arbuscular endomycorrhizal fungi colonizing roots. Appl. Environ. Microbiol. 58, 291-295.

Smit, E., Leeflang, P., Glandorf, B., van Elsas, J. D., and Wernars, K. (1999). Analysis of fungal diversity in the wheat rhizosphere by sequencing of cloned PCR-amplified genes encoding $18 \mathrm{~S}$ rRNA and temperature gradient gel electrophoresis. Appl. Environ. Microbiol. 65, 2614-2621.

Sokol, H., Leducq, V., Aschard, H., Pham, H. P., Jegou, S., Landman, C., et al. (2016). Fungal microbiota dysbiosis in IBD. Gut. doi: 10.1136/gutjnl-2015-310746. [Epub ahead of print].

Stackebrandt, E., and Goebel, B. M. (1994). Taxonomic note: a place for DNA-DNA reassociation and 16S rRNA sequence analysis in the present species definition in bacteriology. Int. J. Syst. Evol. Microbiol. 44, 846-849. doi: 10.1099/00207713-44-4-846

Tanabe, Y., Watanabe, M. M., and Sugiyama, J. (2002). Are Microsporidia really related to Fungi? a reappraisal based on additional gene sequences from basal fungi. Mycol. Res. 106, 1380-1391. doi: 10.1017/S095375620200686X

Tang, J., Iliev, I. D., Brown, J., Underhill, D. M., and Funari, V. A. (2015). Mycobiome: approaches to analysis of intestinal fungi. J. Immunol. Methods 421, 112-121. doi: 10.1016/j.jim.2015.04.004

Taylor, D. L., Hollingsworth, T. N., McFarland, J. W., Lennon, N. J., Nusbaum, C., and Ruess, R. W. (2014). A first comprehensive census of fungi in soil reveals both hyperdiversity and fine-scale niche partitioning. Ecol. Monogr. 84, 3-20. doi: $10.1890 / 12-1693.1$

Tedersoo, L., Bahram, M., Polme, S., Koljalg, U., Yorou, N. S., Wijesundera, R., et al. (2014). Fungal biogeography. Global diversity and geography of soil fungi. Science 346:1256688. doi: 10.1126/science. 1256688

Toju, H., Tanabe, A. S., Yamamoto, S., and Sato, H. (2012). Highcoverage ITS primers for the DNA-based identification of ascomycetes and basidiomycetes in environmental samples. PLOS ONE 7:e40863. doi: 10.1371/journal.pone.0040863

Underhill, D. M., and Iliev, I. D. (2014). The mycobiota: interactions between commensal fungi and the host immune system. Nat. Rev. Immunol. 14, 405-416. doi: 10.1038/nri3684

van Burik, J. A., Schreckhise, R. W., White, T. C., Bowden, R. A., and Myerson, D. (1998). Comparison of six extraction techniques for isolation of DNA from filamentous fungi. Med. Mycol. 36, 299-303. doi: 10.1080/02681219880000471

Wang, Q., Garrity, G. M., Tiedje, J. M., and Cole, J. R. (2007). Naive Bayesian classifier for rapid assignment of rRNA sequences into the new bacterial taxonomy. Appl. Environ. Microbiol. 73, 5261-5267. doi: 10.1128/AEM.00062-07

Westcott, S. L., and Schloss, P. D. (2015). De novo clustering methods outperform reference-based methods for assigning $16 \mathrm{~S}$ rRNA gene sequences to operational taxonomic units. PeerJ 3:e1487. doi: 10.7717/peerj.1487 
Wheeler, M. L., Limon, J. J., Bar, A. S., Leal, C. A., Gargus, M., Tang, J., et al. (2016). Immunological consequences of intestinal fungal dysbiosis. Cell Host Microbe 19, 865-873. doi: 10.1016/j.chom.2016. 05.003

White, T., Bruns, T., Lee, S., and Taylor, J. (1990). "Amplification and direct sequencing of fungal ribosomal RNA genes for phylogenetics," in PCR Protocols: A Guide to Methods and Applications, eds M. Innis, D. Gelfand, J. Sninsky, and T. White (Orlando, FL: Academic Press), 315-322.

Yuille, M., van Ommen, G. J., Brechot, C., Cambon-Thomsen, A., Dagher, G., Landegren, U., et al. (2008). Biobanking for Europe. Brief. Bioinform. 9, 14-24. doi: 10.1093/bib/bbm050
Conflict of Interest Statement: The authors declare that the research was conducted in the absence of any commercial or financial relationships that could be construed as a potential conflict of interest.

Copyright (c) 2017 Halwachs, Madhusudhan, Krause, Nilsson, Moissl-Eichinger, Högenauer, Thallinger and Gorkiewicz. This is an open-access article distributed under the terms of the Creative Commons Attribution License (CC BY). The use, distribution or reproduction in other forums is permitted, provided the original author(s) or licensor are credited and that the original publication in this journal is cited, in accordance with accepted academic practice. No use, distribution or reproduction is permitted which does not comply with these terms. 